\title{
ECOEFICIÊNCIA E PREÇO SOMBRA DAS EMISSÕES DE GASES DE EFEITO ESTUFA NA SUINOCULTURA BRASILEIRA
}

\author{
Pollyanna Araujo Alencar \\ Carlos Rosano-Peña** \\ Patrícia Guarnieri*** \\ André Luiz Marques Serrano****
}

RESUMO: Nas últimas décadas a suinocultura tornou-se uma importante atividade para o agronegócio brasileiro, com contribuições para o desenvolvimento econômico do país. Contudo, esse setor apresenta um grande potencial poluente, com um elevado custo ambiental relacionado ao processo produtivo. Neste contexto, o presente trabalho tem como objetivo estimar a ecoeficiência na produção brasileira de suínos, os preços sombra e os custos totais das emissões de Gases de Efeito Estufa (GEE). O método utilizado para atingir o objetivo proposto foi a Análise Envoltória de Dados combinada com o método de Funções Distâncias Direcionais. Foram estabelecidos dois padrões de dados na pesquisa dos municípios produtores de suínos em nove Estados brasileiros. Para o Padrão 1, em média, os municípios podem elevar a produção em 36,9\% e reduzir as emissões de $\mathrm{CO}_{2}$ e na mesma proporção, com a mesma quantidade de insumos. Para o Padrão 2 esse valor foi de $47,22 \%$. Os preços sombra médios ficaram em $\mathrm{R} \$ 3.539,81$ no Padrão 1 e $\mathrm{R} \$ 3.677,07$ no Padrão 2. As estimativas dos custos totais da redução das emissões de $\mathrm{CO}_{2} \mathrm{e}$ foram de $\mathrm{R} \$ 1,37$ bilhões, o equivalente a 468\% da produção em 2006, para o Padrão 1, e R\$1,42 bilhões, correspondente a 486\% da produção em 2006, para o Padrão 2. Portanto, constatou-se que a incorporação desses custos é praticamente inviável, pois levaria à descontinuidade das propriedades. Desse modo, a pesquisa indica a necessidade de redesenhar um sistema dissuasivo de punições e incentivos, que considere os preços sombra para que os produtores de suínos internalizem as externalidades negativas e busquem compensações para as ações que reduzam as emissões ou utilizem tecnologias mais limpas. A principal contribuição do trabalho é a inclusão da análise de eficiência considerando a variável ambiental, questão ainda pouco abordada na literatura.

\footnotetext{
Mestre em Agronegócio pela Universidade de Brasília (UnB). Brasil. E-mail: polly.anna@bol.com.br

** Doutor em Economia pela Universidade Russa Amizade dos Povos. Docente associado do Departamento de Administração e nos Programas de pós-graduação em agronegócio e economia da Universidade de Brasília (UnB). Brasil.

${ }^{* * *}$ Doutora em Engenharia de Produção pela UFPE. Docente no Programa de Pós-Graduação em Agronegócio e do Programa de Pós-Graduação em Administração da Universidade de Brasília (UnB). Brasil.

${ }^{* * * *}$ Doutor em Economia pela Universidade de Brasília (UnB). Docente Adjunto do Departamento de Engenharia de Produção, Faculdade de Tecnologia (FT) e da Pós Graduação em Ciências Contábeis (FACE). Brasil.
} 
PALAVRAS-CHAVE: Análise envoltória de dados; Ecoeficiência; Gases de efeito estufa; Preço sombra; Suinocultura.

\title{
ECO-EFFICIENCY AND SHADOW PRICE OF GREENHOUSE GAS EMISSIONS IN SWINE CULTURE IN BRAZIL
}

\begin{abstract}
Swine breeding has recently become an important activity for agribusiness in Brazil, with great contributions for the country's economic development. However, the activity features immense pollution potential, with high environmental costs for the production process. Current study estimates the eco-efficiency in the production of swine, shadow price and total costs of greenhouse gas emissions (GGE) by Data Envelopment Analysis plus Directional Distance Functions. Two data standards of the municipalities with swine production in nine Brazilian states were established. In the case of Standard 1, the municipalities may have a $36.93 \%$ production rise and reduce $\mathrm{CO}_{2}$ at the same rate, with the same amount of inputs. In the case of Standard 2, production rise was $47.22 \%$. Mean shadow prices reached $\mathrm{R} \$$ 3,539.81 for Standard 1 and $R \$ 3,677.07$ for Standard 2. Estimates of total costs for $\mathrm{CO}_{2}$ emission reduction were $\mathrm{R} \$ 1.37$ billion, equivalent to $468 \%$ of production in 2006 , for Standard 1, and $\mathrm{R} \$ 1.42$ billion, equivalent to $486 \%$ of production in 2006 , for Standard 2. Incorporation of costs was practically unfeasible since it would lead to quittance of the activity. Research shows the need for re-designing a dissuasive system of punishments and incentives for shadow prices so that swine producers become aware of the negative externalities and seek compensations for activities that reduce emission or employ cleaner technologies. The assay's main contribution is the inclusion of efficiency analysis with environmental variable, an issue not dealt with in the literature.
\end{abstract}

KEY WORDS: Data envelopment analysis; Eco-efficiency; greenhouse gases; shadow price; Swine culture.

\section{INTRODUÇÃO}

A expansão da agricultura e da pecuária foi um dos principais fatores de transformação da ecosfera, e do aumento populacional da humanidade nas últimas décadas (MAZOYER; ROUDART, 2010). A domesticação de suínos acompanhou a história da civilização. Inicialmente, os suínos e outros animais eram transportados vi- 
vos para abate durante grandes viagens, pois não havia tecnologia para conservação da carne. Por serem animais onívoros, ou seja, se alimentam tanto de carne quanto vegetais, tal fato contribuiu para sua domesticação (GASTARDELO; MELZ, 2014).

A carne de porco permanece como a mais consumida no mundo e sua oferta aumenta ano a ano, acompanhando o crescimento da população mundial. O Departamento de Agricultura dos Estados Unidos (2015) estima que a suinocultura seja responsável por $43 \%$ do consumo global de carnes, sendo que a produção mundial cresceu por volta de $20 \%$ no período 2004-2014, atingindo o volume de 110.476 mil toneladas (DEPARTAMENTO DE AGRICULTURA DOS ESTADOS UNIDOS - USDA, 2015).

Entretanto, o impacto que o avanço da produção agropecuária tem exercido sobre o meio ambiente vem aumentando a preocupação da sociedade com as mudanças do clima no planeta e o risco a respeito da sobrevivência de gerações futuras. Dentre os principais impactos ambientais causados pela suinocultura, com destaque para a poluição das águas superficiais e subterrâneas, estão a alteração das características químicas, físicas e biológicas do solo, a poluição do ar pela emissão de gases de efeito estufa, tendo como principais o $\mathrm{NH}_{3}, \mathrm{CO}_{2}, \mathrm{CH}_{4}, \mathrm{~N}_{2} \mathrm{O}$ e $\mathrm{H}_{2} \mathrm{~S}$ (SARDÁ et al., 2010).

O crescimento sustentável da produção de alimentos é um dos principais desafios para o desenvolvimento futuro da humanidade. Em outras palavras, se por um lado é preciso aumentar a produção agropecuária, por outro, é necessário controlar e reduzir o impacto ambiental. Logo, é possível internalizar as externalidades associadas ao processo produtivo da suinocultura através da modelagem das tecnologias de produção multioutputs?

As primeiras pesquisas que contemplaram as questões ambientais utilizaram Análise Envoltória de Dados, Data Envelopment Analysis - DEA na língua inglesa, no âmbito das técnicas de fronteiras eficientes, nas quais modelaram os outputs ambientais indesejáveis ora como inputs a minimizar, ora como variáveis outputs com valores negativos ou invertidos a maximizar, usando as medidas de eficiência radiais orientadas de Farrell (1957) (ZHOU et al., 2008). Entretanto, um dos grandes avanços teóricos nessa área foi a introdução, por Chung et al. (1997), de funções distâncias direcionais multiobjetivas - FDDM, que permitiram medir o de- 
sempenho ambiental de unidades produtivas em termos de capacidade de expansão da produção, com a redução simultânea dos insumos e dos produtos indesejados. Ressalta-se o trabalho de Arandia e Aldanondo-Ochoa (2011), que compararam os impactos ambientais entre fazendas de agricultura convencional e de agricultura orgânica, e avaliaram o esforço que a inclusão desses impactos causaria nas fazendas seja da agricultura convencional, seja na agricultura orgânica. Ainda nesse contexto, Salnykov e Zelenyuk (2005) calcularam preços sombras das emissões de dióxido de carbono, dióxido de enxofre e óxido de nitrogênio para uma amostra de países.

$\mathrm{Na}$ literatura brasileira também existem estudos que avaliam a eficiência agropecuária brasileira com as técnicas DEA, onde é possível citar Gomes (2004), Magalhães e Campos (2006), Gonçalves et al. (2006), Gomes et al. (2009), Campos et al. (2014) e Souza (2014). Porém, observa-se que o emprego específico do DEA em estudos relacionados à ecoeficiência da agricultura brasileira e de sustentabilidade no geral ainda é incipiente, podendo ser citados apenas os trabalhos de Padrão et al. (2012), que estimam a eficiência ambiental DEA e o custo de oportunidade do Código Florestal para a Amazônia, e de Rosano-Peña et al. (2014), que avaliam a ecoeficiência e a sustentabilidade da agricultura brasileira em geral usando funções distância direcionais com DEA.

Com o objetivo de estimar a ecoeficiência da produção brasileira de suínos, os preços sombra e o custo de oportunidade das emissões de gases de efeito estufa (GEE) do setor, nos municípios dos Estados do Ceará, Goiás, Mato Grosso, Mato Grosso do Sul, Minas Gerais, São Paulo, Paraná, Santa Catarina e Rio Grande do Sul, esta pesquisa utilizou a Análise Envoltória de Dados (DEA) combinada com o método de funções distâncias direcionais (FDD) para calcular as emissões de GEE da suinocultura dos municípios que realizaram abates de suínos, computou-se os índices de ecoeficiência e as melhorias necessárias para que os municípios ecoineficientes tornem-se ecoeficientes, e calculou-se o preço sombra e os custos de oportunidade das emissões de GEEs nos municípios avaliados. 


\section{REVISÃO DA LITERATURA}

\subsection{SUINOCULTURA BRASILEIRA}

A produção suína brasileira passou por mudanças significativas, seja no aumento de escala e especialização, quanto na concentração geográfica, o que proporcionou a expansão da integração entre suinocultores e agroindústria, com ganhos econômicos e de eficiência (COSER, 2010).

Em 1940, o rebanho suíno brasileiro era de 23,8 milhões de animais, e a zona produtiva estava concentrada no cinturão de produção de milho nos Estados da região Sul e Sudeste. Já nos anos seguintes ocorreu a expansão da suinocultura em outras regiões, evidenciando-se um crescimento da produção na região CentroOeste. Região com altos índices de crescimento, especialmente pelas condições associadas à produção de grãos, abundância de água e clima favorável, possibilitando a redução dos custos de produção (ASSOCIAÇÃO BRASILEIRA DOS CRIADORES DE SUÍNOS - ABCS; MINISTÉRIO DA AGRICULTURA, PECUÁRIA E ABASTECIMENTO - MAPA; EMPRESA BRASILEIRA DE PESQUISA AGROPECUÁRIA - EMBRAPA, 2011; SANTOS; TALAMINI; MARTINS, 2011).

No ano de 2011, a produção suinícola estava concentrada nos Estados de Santa Catarina (25,38\%), Rio Grande do Sul (21,58\%), Paraná (18,64\%), Minas Gerais (13,40\%), São Paulo (5,17\%), Mato Grosso (3,99\%), Goiás (3,72\%), e Mato Grosso do Sul (2,79\%), respondendo pela quase totalidade dos abates de suínos inspecionados no país. Os Estados das regiões Norte e Nordeste não demonstraram crescimento, pois a escassez e o alto preço dos insumos inviabilizam a expansão da atividade nestas regiões (INSTITUTO BRASILEIRO DE GEOGRAFIA E ESTATÍSTICA IBGE, 2011; MIELE et al., 2013).

Segundo o MAPA (2015), este setor tem um maior percentual de crescimento no segmento brasileiro de carnes nas projeções para o período de 2015 a 2025 , com taxas estimadas de crescimento de $35,1 \%$, superando a carne de frango $(34,7 \%)$ e da carne bovina $(23,3 \%)$.

Porém, as formas intensivas de produção resultaram em um aumento do potencial poluidor, principalmente nas regiões com grande concentração desta atividade produtiva no país (MIELE, 2006). 
De acordo com Silva, Bassi e Nascimento (2011) até os anos de 1970, os dejetos de suínos não constituíam um fator de preocupação, já que a baixa produção destes era utilizada como adubo. Contudo, a partir da adoção do sistema de produção com grande concentração de animais confinados em pequenas áreas, ocasionou o aumento do volume de dejetos, causando impacto ambiental. Os autores ressaltam que um dos fatores agravantes da grande produção dos dejetos de suínos é a contribuição para o aquecimento global, pois durante o processo de decomposição anaeróbica desses dejetos ocorre a produção do biogás, composto principalmente por metano $\left(\mathrm{CH}_{4}\right)$, que é um gás com potencial de aquecimento 21 vezes maior que o dióxido de carbono $\left(\mathrm{CO}_{2}\right)$.

Portanto, a suinocultura brasileira precisa atender às exigências tanto do mercado quanto do próprio ecossistema, que cada vez mais exige uma produção sustentável, com um padrão crescente de qualidade, com rastreabilidade, que proporcione segurança alimentar e ambiental em atendimento às expectativas do consumidor (FÁVERO et al., 2003).

Tendo o propósito de atingir esses objetivos, é preciso aplicar diretrizes de boas práticas que enfatizem uma exploração econômica de suínos viável, de forma a preservar o meio ambiente e a justiça social, com a manutenção da produtividade. Fávero et al. (2003) frisam que as boas práticas agropecuárias podem ser utilizadas por produtores em qualquer escala de produção, por contemplarem todas as etapas de produção.

\subsection{EFICIÊNCIA E ECOEFICIÊNCIA}

Além dos conceitos tradicionais da produtividade parcial da terra e do trabalho, o indicador mais popular para avaliação da competitividade agropecuária é o termo eficiência.

De um modo geral, na literatura especializada, a eficiência técnica é caracterizada como a capacidade de uma unidade produtiva aumentar a produção, com um dado valor de insumos ou de reduzir os insumos necessários para atingir um nível dado de produção (ROSANO-PEÑA, 2008). Nesse ponto, Rosano-Peña (2008) infere como conceito de eficiência técnica-ambiental, também chamada de ecoeficiência, a 
capacidade que uma unidade produtiva tem de fornecer produtos desejados com o menor nível de insumos e impactos ambientais.

Callens e Tyteca (1999) enfatizam que a ecoeficiência é condição necessária para alcançar a sustentabilidade, mas não suficiente, pois os valores mínimos dos impactos ambientais são definidos em relação a uma amostra e não representam a capacidade do planeta de absorver os impactos ambientais.

Segundo Zhang et al. (2008), o termo ecoeficiência remonta à década de 1970, tendo sido utilizado por Freeman et al. (1973) como um indicador da eficiência ambiental. Posteriormente, esse conceito foi difundido pelo World Business Council for Sustainable Development (WBCSD, 1992). Conforme explicita esse documento, elaborado em 1992, para que um processo produtivo seja considerado ecoeficiente é preciso que tenha preços competitivos, satisfaça as necessidades humanas, proporcione qualidade de vida, consuma recursos naturais de forma reduzida e respeite a capacidade de sustentação do planeta.

\subsection{VALORAÇÃO ECONÔMICA AMBIENTAL}

Neste trabalho, a relação entre as atividades humanas (suinocultura) e o meio ambiente é enfatizada através da produção de dejetos que tem alto potencial de poluição do ar, da água e do solo.

Mota (2001) esclarece que as mercadorias (bens econômicos) têm valor econômico, ou seja, preços determinados pelo mercado, já os recursos do ecossistema, como florestas públicas, mar e o ar não são mercadorias, não têm seus preços fixados pelo mercado, pois estes recursos naturais são considerados ativos de livre acesso a todos.

Segundo Castro (2015), diversos métodos foram desenvolvidos na tentativa de aperfeiçoamento da mensuração monetária do meio ambiente, contudo, nenhum método é completamente apropriado na avaliação de um ativo ambiental. Uma característica particular dessa valoração é a divisão dos métodos por presença ou não da função demanda em seu arcabouço teórico.

A divisão dos métodos de valoração por presença ou não da Função pode ser: 
- Presença da Função demanda: Método de Valoração Contingente (MVC), Método Custos de Viagem (MCV) e Método de Preços Hedôni$\cos (\mathrm{MPH})$;

- Não contemplam a Função demanda: Método Dose-Resposta (MDR), Método Custo de Reposição (MCR), Método Custo de Oportunidade (MCO) e Método Custos Evitados (MCE) (CASTRO, 2015).

Os métodos com a presença da função de demanda têm como meta revelar ou obter as preferências dos indivíduos, ou seja, admitem que mudanças no ecossistema e na disponibilidade dos recursos naturais alteram o nível de bem-estar dos consumidores e, portanto, é possível identificar uma medida de disposição a pagar relacionadas a essas mudanças. Já os métodos que não contemplam a função demanda (utilizado neste trabalho) estão reunidos em outro grupo que privilegia a função produção, pois caracterizam os recursos naturais como insumos e o impacto ambiental como subprodutos, também chamados de externalidades que podem ter natureza negativa ou natureza positiva. A quantificação da contribuição desse recurso no processo produtivo é estimada através de preços sombra ou custo de oportunidade (CASTRO, 2015).

No quadro conceitual da ecoeficiência, a valoração é interpretada como um conflito de escolhas (trade-off) entre o incremento da produção de produtos desejados e a redução da contaminação ambiental que não tem preço de mercado. Esse valor é chamado de preço sombra (implícito) que corresponde ao custo de oportunidade de uma atividade, à perda causada pela oportunidade renunciada, ou seja, aos benefícios que poderiam ser obtidos a partir dessa alternativa abandonada. Portanto, o preço sombra pode ser estimado pela receita ou lucro a ser renunciada para restringir a degradação ambiental (CATALÁN; DESTÉFANO, 2011). 


\section{ASPECTOS METODOLÓGICOS}

A presente pesquisa é aplicada, possui caráter quantitativo, com fontes de dados secundárias. O método utilizado e as variáveis são caracterizados a seguir.

\subsection{QUANTIFICAÇÃO DA EFICIÊNCIA E DA ECOEFICIÊNCIA}

Para quantificar o conceito de eficiência e ecoeficiência basicamente utilizam-se os métodos de fronteira. Essas técnicas podem-se dividir em dois grupos: i) os paramétricos, que buscam calcular a eficiência através de uma função de produção que relacione as entradas e saídas; e ii) os não-paramétricos, que utilizam funções distância de Shephard (1953) para calcular a eficiência a partir da construção de uma fronteira eficiente, formada pelas unidades que possuem as melhores práticas, considerando a relação ótima entre entradas e saídas (MARIANO, 2008).

Tyteca (1996), Scheel (2001) e Zhou et al. (2008) realizaram revisões fundamentadas sobre as abordagens utilizadas na mensuração da eficiência e ecoeficiência. Segundo estes autores, o método paramétrico mais utilizado na literatura é a Análise das Fronteiras Estocásticas (Stochastic Frontier Analysis - SFA), que parte de uma relação funcional, a priori, definida entre insumos e produtos, mas é pouco utilizada na análise da ecoeficiência pela dificuldade de incluir na análise os produtos indesejados (externalidades) sem preço de mercado e de criar um valor agregado da produção. Esse método utiliza programação linear ou máxima verossimilhança. No que se refere aos métodos não paramétricos, o mais usado é o DEA. O objetivo básico do DEA consiste em comparar unidades produtivas (Decision Making Unit - DMU) que realizam tarefas similares e se diferenciam apenas nas quantidades de inputs que consomem e de outputs que produzem. Esse método utiliza programação linear para avaliar a eficiência relativa de DMUs considerando os múltiplos insumos necessários para gerar múltiplos produtos, podendo internalizar as externalidades associadas ao processo produtivo. 
3.2 ECOEFICIÊNCIA COM O MÉTODO ANÁLISE ENVOLTÓRIA DE DADOS E FUNÇÕES DISTÂNCIA DIRECIONAIS

Para estimar a ecoeficiência de cada unidade produtiva Chung et al. (1997) começam com a caracterização da tecnologia ecoeficiente. A forma genérica de descrevê-la é por meio do Conjunto de Possibilidade de Produção (CPP). Esse conjunto incorpora os vetores inputs utilizados ( $\mathrm{x} \in R_{+}^{n}$ ) na produção de todos os outputs (u $\in R_{+}^{m}$ ) nas $i$ unidades tomadoras de decisão observadas (Decision Making Units $D M U s)$ em um determinado período de tempo. Aqui $\mathbf{u}=(\mathbf{y}, \mathbf{b}), \mathbf{y} \in R_{+}^{p}$ é o subvetor de outputs desejados, b $\in \boldsymbol{R}_{+}^{q}$ é o dos indesejados e $\mathrm{m}=\mathrm{p}+\mathrm{q}$. Formalmente, $\mathrm{CPP}=\{(\mathrm{x}, \mathrm{y}, \mathrm{b}): \mathrm{x}$ pode produzir $\mathrm{y}, \mathrm{b} \wedge \mathrm{x}, \mathrm{y}, \mathrm{b} \geq 0\}$.

Além das propriedades clássicas formuladas por Grosskopf (1986), esse CPP deve-se cumprir três outras adicionais, registradas por Picazo-Tadeo et al. (2012):

i. As atividades econômicas sempre geram impactos no ecossistema, de tal forma que a única alternativa de não poluir o ambiente é não produzir. Ou seja, ambos os outputs são complementares e não existem tecnologias 100\% limpas. Essa propriedade é chamada null jointness.

ii. Existindo ecoeficiência, a redução dos dois tipos de outputs é possível, mas a eliminação isolada dos produtos indesejáveis é impossível. Isto significa que a eliminação de poluentes envolve uma compensação (trade-off), um custo medido em termos de oportunidades, como o valor em que deve ser reduzido o produto (y) máximo possível (com os recursos disponíveis) para mitigar o impacto ambiental. Esse valor é chamado de preço sombra e representa o custo da degradação ambiental para o produtor (FÄRE; GROSSKOPF, 1996). Esta propriedade é denominada descarte fraco de produtos indesejados.

iii. Havendo ecoineficiência, é possível gerar uma quantidade maior de poluentes $(b)$ com a mesma quantidade de y e x. Portanto, a redução do output indesejável não implica a diminuição do produto desejável, sendo o custo de oportunidade da redução do impacto ambiental nulo neste caso. Esta propriedade é nomeada descarte forte de produtos desejados. 
Para Picazo-Tadeo et al. (2012), formalmente, supondo rendimentos constantes de escala e descarte forte de outputs desejáveis, o CPP que satisfaz o axioma de descarte fraco dos outputs indesejáveis é:

$$
\mathrm{CPP}=\left\{(\mathrm{x}, \mathrm{y}, \mathrm{b}) \epsilon R_{+}^{n+p+q}: X z \leq x, Y z \geq y, B z=b, z \in R_{+}^{k}\right\}
$$
onde z é o vetor de intensidade de cada DMU na definição do hiperplano de referência, resultado de combinações lineais das melhores práticas; $\mathrm{x}=\left(x_{1}, x^{2}, \ldots\right.$, $\left.x_{n}\right)$ input utilizado para produzir o vetor $\mathrm{y}=\left(y_{1}, y_{2}, \ldots, \mathrm{y}_{\mathrm{p}}\right)$ e o vetor $\mathrm{b}=\left(b_{1}\right.$, $\left.b x_{2}, \ldots, b_{q}\right) . \mathrm{X}_{(n x k)}, \mathrm{Y}_{(p x k)}$ e $\mathrm{B}_{(q x k)}$ representam as matrizes inputs, outputs desejados e outputs indesejados, respectivamente, da amostra das k DMUs.

Para calcular ecoeficiência de cada DMU, Chung, Färe e Grosskopf (1997) introduzem o conceito de Função Distância Direcional (Directional Distance Function - DDF):

$$
\vec{D}=\left(\mathrm{x}, \mathrm{y}, \mathrm{b} ;-g_{x}, g_{y},-g_{b}\right)=\operatorname{Sup}\left\{\beta:\left(\mathrm{x}-\beta g_{x}, \mathrm{y}+\beta g_{y}, \mathrm{~b}-\beta g_{b}\right) \in \mathrm{CPP}\right\}
$$

$O$ valor ótimo de $\beta$ deve ser maior ou igual a zero. Se $\beta=0$, a unidade avaliada é ecoeficiente, não pode incrementar os produtos desejáveis e reduzir simultaneamente os insumos e subprodutos indesejáveis com a tecnologia disponível; se $\beta 0$ é ecoineficiente. Assim, $\beta$ indicará o percentual em que a DMU avaliada poderia incrementar todos os produtos desejáveis e reduzir simultaneamente os insumos e subprodutos indesejáveis quando a direção a priori definida pelo pesquisador - tomador de decisão, a partir do vetor direção, é (,

Para cada $\mathrm{DMU}^{\mathrm{i}}, \beta$ calcula-se resolvendo o problema de programação linear (PPL) (2).

$$
\mathrm{D}=\left(\mathrm{x}, \mathrm{y}, \mathrm{b} ;-\mathrm{g}_{\mathrm{x}}, \mathrm{g}_{\mathrm{x}},-\mathrm{g}_{\mathrm{b}}\right)=\operatorname{Max} \beta^{\mathrm{k}}
$$

s.a:

$$
\begin{aligned}
& (1+\beta \mathrm{gy}) * \mathrm{y}^{\mathrm{k}} \leq Y z \\
& (1-\beta \mathrm{gy}) * \mathrm{~b}^{\mathrm{k}}=Y z \\
& (1-\beta \mathrm{gy}) * \mathrm{x}^{\mathrm{k}} \leq Y z \\
& \mathrm{z} \leq 0
\end{aligned}
$$


é o vetor de intensidade estimado de cada DMU na definição do hiperplano de referência, resultado de combinações lineais das melhores práticas e seu equivalente no PPL dual mostra o preço sombra de cada input e cada output na determinação da ecoeficiência;

A medida de eficiência (2), além de considerar a existência de descarte fraco dos produtos indesejáveis e o descarte forte dos produtos desejáveis e entradas, considera a existência de retornos constantes de escala. A determinação dos rendimentos variáveis de escala em PPL (2) exige apenas a inclusão da restrição $\sum \mathrm{z}=1$.

Para Rosano-Peña et al. (2013), no PPL (1) para cada vetor direcional estabelecido a priori, dependendo dos objetivos do tomador de decisão, as funções distância direcionais permitem calcular diferentes medidas de eficiência, que projetam as unidades ecoineficientes na fronteira, satisfazendo o conceito ótimo de Pareto (ou seja, quando é impossível com a tecnologia dada melhorar a situação de uma dimensão sem degradar a situacão de qualquer outra). Neste trabalho usa-se o vetor direção $\quad\left(-g_{x}=0, g_{y}=1,-g_{b}=1\right)$, que busca maximizar $y$ e minimizar b com vetores fixos de $x$.

Segundo Picazo-Tadeo et al. (2012), o significado do método de funções distância e do pode ser ilustrado graficamente. Suponha-se que as DMU's avaliadas (A, B, C, D, E, F e H), utilizando uma determinada quantidade de insumos, produzem um output desejável (y) e um output indesejável (b), representados nos eixos vertical e horizontal respectivamente. Dessa forma, na Figura 1, a área $\mathrm{OABCDH}$ representa o conjunto de possibilidade de produção cuja fronteira eficiente é formada pelo segmento $\overline{O A B C D}$. 


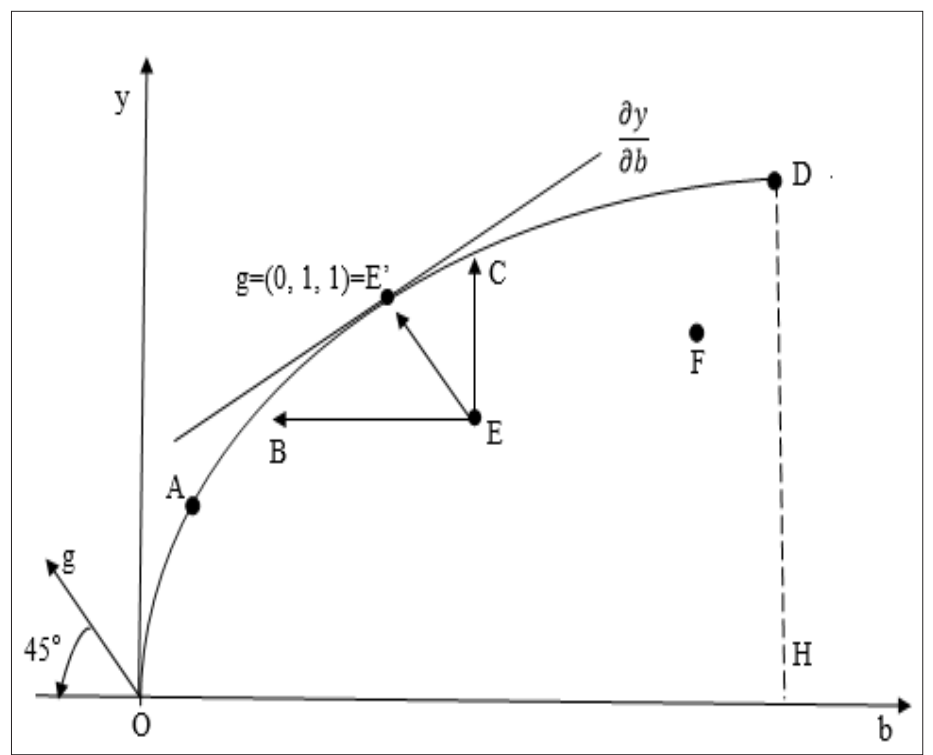

Figura 1. Exemplificação das Funções Distância Direcionais Fonte: Elaborado pelos autores com base em Picazo-Tadeo et al. (2012).

Assim, E (como F e H) é ecoineficiente. Seu nível de ineficiência e a projeção na fronteira dependem do vetor direção definido a priori. Por exemplo, desejando saber em quanto pode ser acrescentado o output desejado de E com o mesmo nível de impacto ambiental e insumos, ou seja, determinando $g=\left(-g_{\mathrm{x}}=0, g_{\mathrm{v}}=1\right.$, $\left.g_{\mathrm{b}=} 0\right)$, o PPL (2) projetará E no ponto $\mathrm{C}=\left(b^{c}, y^{c}\right)=\left[b^{E}, y^{E}\left(1+\beta g_{y}\right)\right]$. No entanto, aspirando reduzir o impacto ambiental mantendo o produto desejado e os insumos constantes, usando o vetor direção $g=\left(-g_{\mathrm{x}}=0, g_{\mathrm{y}}=0,-g_{\mathrm{b}=} 1\right)$, o PPL (2) projetará E no ponto $\mathrm{B}=\left(b^{B}, y^{B}\right)=\left[b^{E}\left(1-\beta g_{y}\right), y^{E}\right]$. Por fim, querendo aumentar $y$ e reduzir $b$ simultaneamente com os mesmo insumos, utilizando $g=\left(-g_{\mathrm{x}}=0, g_{\mathrm{y}}=1, g_{\mathrm{b}}=1\right), \mathrm{o}$ PPL (2) projetará E no ponto E' $=\left[b^{F}(1-\right.$ $\left.\left.\beta g_{b}\right), y^{F}\left(1+\beta g_{b}\right)\right]$. Portanto, observa-se que, satisfazendo o conceito ótimo de Pareto, é possível aumentar de diferentes formas a ecoeficiência. 


\subsection{ESTIMATIVA DOS PREÇOS SOMBRA}

Como já foi mencionado anteriormente, a produção de bens e serviços desejáveis gera efeitos negativos ao meio ambiente. A redução desses produtos indesejáveis (bad outputs) tem um custo, ou seja, a eliminação desses poluentes envolve uma compensação (trade-off), um custo medido em termos de oportunidades, como o valor em que deve ser reduzido o produto (y) máximo possível (com os recursos disponíveis) para mitigar o impacto ambiental. Esse valor é chamado de preço sombra e representa o custo da degradação ambiental para o produtor (FÄRE et al., 1996; FÄRE et al., 2006; ARANDIA; ALDANONDO-OCHOA, 2011).

$O$ preço sombra representa a taxa marginal de transformação de um bem em outro e mede o custo de oportunidade (marginal) de um bem em termos do outro. Assim, pode nesta pesquisa representar o custo marginal da redução das emissões de carbono equivalente oriundas dos abates de suínos.

Na Figura 1, observa-se a tangente na projeção E' da fronteira do CPP. A inclinação dessa reta representa taxa marginal de transformação (TMgT) entre os produtos desejados $(y)$ e os subprodutos indesejados $(b)$ indicando o custo de oportunidade da produção de $b$ em relação ao output y, ou seja, quanto do produto desejado y se deixa de produzir para se reduzir, com os mesmos insumos, uma quantidade adicional do subproduto b (COGGINS; SWINTON, 1996).

Para calcular as TMgT segue-se um procedimento. Inicialmente, calculam-se ecoeficiência para todas as unidades produtivas avaliadas. Em seguida corrigem-se as ineficiências das unidades ineficientes, projetando-as para a fronteira com o vetor direcional $(0,1,1)$. A projeção será representada por um ponto na fronteira eficiente, que terá, para o caso de $\mathrm{DMU}^{\mathrm{E}}$, a seguinte coordenada $\left[\mathrm{bE}\left(1-\beta g_{b}\right) ; \mathrm{y}^{\mathrm{E}}(1+\right.$ $\left.\beta g_{b}\right)$ ]. Posteriormente, considerando-se as projeções de todas as DMUs avaliadas, resolve-se novamente PPL (2). O dual do (2) permite estimar o preço sombra ou multiplicador de Lagrange $(\lambda)$ para cada uma das pressões ambientais e dos produtos desejáveis. Ele mostra a variação na função objetivo decorrente de uma variação marginal dos produtos, o que permite estimar a variação relativa do produto desejado decorrente de uma variação marginal do produto indesejado mantendo o nível de ecoeficiência máximo. Segundo Oude-Lansink e Silva (2004), a taxa marginal de transformação de cada $\mathrm{DMU}^{\mathrm{k}}$ é: 


$$
\operatorname{TMgT}_{y b}{ }^{k}=\frac{\partial y}{\partial b}=\frac{\partial \beta^{k}}{\partial b^{k}} / \frac{\partial \beta^{k}}{\partial y^{k}}=\frac{\lambda_{b}{ }^{k}}{\lambda_{y}{ }^{k}}
$$

Desta forma, se conhecemos o preço de mercado do produto desejável $\left(\mathrm{P}_{\mathrm{y}}\right)$ e aplicamos o princípio da alocação ótima se obtém o preço sombra de $b$ de cada $\mathrm{DMU}^{\mathrm{k}}$ da seguinte forma:

$$
\mathrm{P}_{\mathrm{b}} \mathrm{TMgT}^{\mathrm{K}} *(\mathrm{Py})
$$

\subsection{GASES DE EFEITO ESTUFA E SUINOCULTURA}

Para o Painel Intergovernamental sobre Mudanças Climáticas (Intergovernmental Panel on Climate Change - IPCC, 1995) os Gases de Efeito Estufa (GEEs) são decorrentes da atividade antrópica e responsáveis por impedirem que a radiação solar refletida no planeta se dissipe na atmosfera, causando aquecimento. O IPCC divide as emissões dos GEE por setores: Energia, Processos Industriais e Uso de Produtos, Agricultura, Florestas e Outros Usos da Terra e Resíduos.

O setor Agropecuário inclui todas as emissões referentes à fermentação entérica, manejo de dejetos de animais, cultivo de arroz, queima de resíduos agrícolas, solos agrícolas, calagem e outros. De acordo com o Segundo Inventário Brasileiro de Emissões Antrópicas de Gases de Efeito Estufa (MCT, 2010) e o IPCC, os principais gases emitidos na suinocultura são o Metano $\left(\mathrm{CH}_{4}\right)$ e Óxido Nitroso $\left(\mathrm{N}_{2} \mathrm{O}\right)$.

$\mathrm{E}_{\mathrm{FE}}=\mathrm{PA} \times \mathrm{FE} \times 10^{-9}$

Onde:

$\mathrm{E}_{\mathrm{FE}}=$ Emissão da Fermentação Entérica [Gg CH4]

$\mathrm{PA}=$ População Animal [cabeças]

$\mathrm{FE}=$ Fator de emissão para a categoria de animal [g/CH4/animal/ano $]$ 
Devido à necessidade de detalhamento dos dados, para determinar o Fator de Emissão (FE) das emissões de metano provenientes dos suínos, neste trabalho foram utilizados os valores dos parâmetros zootécnicos da suinocultura apresentados no Relatório de Referência do Segundo Inventário Brasileiro de Emissões e Remoções Antrópicas de Gases de Efeito Estufa (MCT, 2010).

Segundo o MCT (2010), o IPCC, através das Diretrizes para Inventários Nacionais de GEE (Guidelines for National Greenbouse Gas Inventories, 1996), estabelece que as estimativas de fermentação entérica da suinocultura, dados em quilos de metano emitido por cabeça ao ano (kg CH4/cabeça/ano) dos municípios pesquisados, toma como parâmetro o Fator de Emissão default (Abordagem Tier 1) no valor do $\mathrm{FE}=1$.

Já para o cálculo de emissões de metano pelo manejo de dejetos de animais baseia-se na mesma metodologia (MCT, 2010) e é dado pela seguinte equação:

$$
\mathrm{E}_{\mathrm{MDA}}=\operatorname{PA} \times \mathrm{FE} \times 10^{-9}
$$

Onde:

$\mathrm{E}_{\mathrm{MDA}}=$ Emissão do Manejo de Dejetos Animais $\left(\mathrm{Gg} \mathrm{CH}_{4}\right)$

$\mathrm{PA}=$ População Animal (cabeças)

$\mathrm{FE}=$ Fator de emissão para a categoria de animal (g/CH4/animal/ano)

A base principal das estimativas dos sistemas de tratamento de dejetos foi o Censo Agropecuário de 2006 (IBGE, 2009). Conforme a metodologia utilizada no Relatório de Referência do MCT (2010), nesta pesquisa também foram considerados os dados de tamanho do rebanho para pequenas e médias propriedades, abaixo de 300 animais, e grandes propriedades, acima de 300 animais.

De forma análoga, foi considerada para o rebanho de suínos em cada município a mesma classificação para pequenos e médios, abaixo de 300 animais, e grandes, acima de 300. A Tabela 1 apresenta os valores dos Fatores de Emissão no Manejo de Dejetos de suínos (Tier 2), conforme os dados do Relatório de Referência do MCT (2010), utilizados nas estimativas das emissões de metano oriundas do manejo de dejetos de suínos. 
Tabela 1. Fatores de emissão de metano para manejo de dejetos de suínos nos Estados pesquisados

\begin{tabular}{l|c}
\hline Estado & $\begin{array}{c}\text { Fator de Emissão Manejo de } \\
\text { Dejetos (kg CH4/cab/ano) }\end{array}$ \\
\hline Ceará & 1,1 \\
\hline Minas Gerais - pequenas propriedades & 2,5 \\
\hline MG - grandes propriedades & 8,6 \\
\hline São Paulo - pequenas propriedades & 2,2 \\
\hline SP - grandes propriedades & 6,4 \\
\hline Paraná - pequenas propriedades & 3,6 \\
\hline PR - grandes propriedades & 6,0 \\
\hline Santa Catarina - pequenas propriedades & 5,8 \\
\hline SC - grandes propriedades & 6,5 \\
\hline Rio Grande do Sul - pequenas propriedades & 3,9 \\
\hline RS - grandes propriedades & 10,0 \\
\hline Mato Grosso do Sul - pequenas propriedades & 2,7 \\
\hline MS - grandes propriedades & 9,7 \\
\hline Mato Grosso - pequenas propriedades & 3,3 \\
\hline MT- grandes propriedades & 8,5 \\
\hline Goiás - pequenas propriedades & 1,9 \\
\hline GO - grandes propriedades & 7,7 \\
\hline
\end{tabular}

Fonte: Adaptado do Segundo Inventário Brasileiro de Emissões Antrópicas de Gases de Efeito Estufa (MCT, 2010).

Ambos os resultados das medições de gás metano, proveniente da fermentação entérica e do manejo de dejetos, foram convertidos em carbono equivalente, pelo fato do gás carbônico ser adotado como métrica internacional para medir os demais GEEs.

O Potencial de Aquecimento Global (GWP) é uma métrica desenvolvida pelo IPCC, para avaliar e comparar os diferentes gases causadores do efeito estufa, levando em conta o potencial de aquecimento global de cada um dos gases. O índice é obtido com base na radiação cumulativa de uma unidade de massa do gás referência, no caso o gás carbônico $\left(\mathrm{CO}_{2}\right)$, projetada em um horizonte de tempo. Isso significa que o $\mathrm{CO}_{2}$ equivalente das emissões é estimado multiplicando o GWP equivalente a quantidade do gás emitido, no caso deste estudo, o metano $\left(\mathrm{CH}_{4}\right)$. O horizonte de 
tempo de referência mais utilizado na literatura é o de 100 anos.

Dessa forma, para encontrar os valores das emissões de gases de efeito estufa municipais dos abates de suínos em Carbono Equivalente, necessário para a estimativa do preço sombra, foram multiplicados os valores encontrados da soma da fermentação entérica e do manejo de dejetos pelo índice GWP de referência do gás metano, que é 21 .

\subsection{VARIÁVEIS E UNIDADES PRODUTIVAS DA PESQUISA}

Com base nos dados do último Censo Agropecuário (IBGE, 2006), os municípios dos Estados do Ceará, Goiás, Mato Grosso, Mato Grosso do Sul, Minas Gerais, São Paulo, Paraná, Santa Catarina e Rio Grande do Sul constituem o conjunto das DMU's avaliadas. Esse conjunto abrange parte significativa do universo da produção suinícola brasileira, com uma representatividade de 56,48\%. Desta forma, foram analisados 3.146 municípios brasileiros. Foram considerados apenas os municípios com dados de abates de suínos, por esta razão, alguns municípios foram eliminados por falta de dados.

A fonte de dado principal para esta pesquisa foi o Censo Agropecuário 2006 (IBGE), por se tratar da investigação mais completa e detalhada sobre o tema, em nível municipal. Concomitantemente, foram utilizados dados da pesquisa sobre o custo da produção suinícola no ano de 2006, realizada conjuntamente pela Companhia Nacional de Abastecimento (CONAB) e Empresa Brasileira de Pesquisa Agropecuária (EMBRAPA) Aves e Suínos (Central de Inteligência de Aves e Suínos) no ano de 2006.

O formato dos dados disponíveis determinou a modelagem de dois modelos ou padrões. O primeiro padrão utilizou as médias anuais estaduais dos custos fixo e variáveis. No entanto, como a utilização dessas médias estaduais para avaliar os municípios deve dar valores aproximados que expressam uma tendência central de um alto nível agregado, construiu-se um novo modelo. Desta forma, o segundo padrão usou as médias anuais municipais dos custos fixos e variáveis estimadas com os dados disponíveis, conforme exposto no Quadro 1: 
Quadro 1. Diferenciação entre as variáveis nos Padrões 1 e 2

\begin{tabular}{|c|c|c|}
\hline \multirow{2}{*}{$\begin{array}{c}\text { ENTRADAS (Inpu- } \\
\text { ts) }\end{array}$} & Padrão 1 & Padrão 2 \\
\cline { 2 - 3 } & $\begin{array}{c}\text { Variáveis de entrada são dadas em } \\
\text { reais por quilos de suínos }(\mathrm{R} \$ / \mathrm{Kg})\end{array}$ & $\begin{array}{c}\text { Variáveis de entrada são dadas em } \\
\text { reais por cabeça de suínos (R\$/ } \\
\text { Cabeça) }\end{array}$ \\
\hline \multirow{2}{*}{ SAÍDAS (Output) } & \multicolumn{2}{|c|}{ Receita dos abates (desejável) } \\
& \multicolumn{2}{|c|}{ Emissões $\mathrm{CO}_{2}$ e dos Abates (indesejável) } \\
\hline
\end{tabular}

Fonte: Elaborado pelos autores.

No Padrão 1, os dados utilizados nas variáveis de entrada não foram alterados. São equivalentes a média anual dos custos fixos e variáveis por Estado.

No Padrão 2, foi necessário estimar os valores das variáveis de entradas, com base na quantidade de suínos abatidos por município. Com esse propósito, foram realizados os seguintes cálculos:

1)Encontrou-se a quantidade de quilos abatidos por município:

$$
\text { Quantidade de quilos abatidos }=\frac{\text { Valor da venda dos abatidos }}{\text { Preço médio do quilo por Estado }}
$$

2)Estimou-se o peso médio do suíno abatido em cada município:

$$
\text { Peso Médio Abatido }=\frac{\text { Quantidade de quilos abatidos }}{\text { Quantidade de cabeças }}
$$

3)A variável custo fixo foi obtida multiplicando o peso médio encontrado em cada município pelo custo fixo médio de cada Estado:

Custo fixo por cabeça $=$ Peso Médio abatidos $\times$ Custo Fixo Médio por Estado

4)O custo variável também foi obtido multiplicando o peso médio encontrado em cada município pelo custo variável médio de cada Estado:

Custo variável por cabeça

$$
\text { = Peso Médio abatidos } \times \text { Custo Variável Médio por Estado }
$$


A variável de saída desejável é dada em reais e representa a receita que cada município obteve com abates de suínos, em 2006.

$\mathrm{Na}$ variável de saída indesejada, dada em toneladas de Carbono Equivalente, representa as emissões de GEE's na produção do rebanho suíno abatido em 2006.

Assim, neste trabalho, são considerados para a análise da ecoeficiência: duas variáveis de entrada (inputs): os custos fixos e os custos variáveis da produção suinícola, dados em reais por quilo; uma variável de saída desejável (desirable output): o valor da produção por abate de suínos, em reais; e uma variável de saída indesejável (undesirable output): representada pela emissão dos gases de efeito estufa por município, medida em toneladas de carbono equivalente (TonCO2eq).

Os resultados de ambos os conjuntos de dados (Padrão 1 e Padrão 2) no modelo DEA com Funções Direcionais e Preço Sombra foram adquiridos com o uso do software MaxDea (versão 6.4). No georreferenciamento dos dados foi utilizado o software IpeaGEO 2.0.

\section{ANÁLISE E DISCUSSÃO DOS RESULTADOS}

A partir da metodologia e dos dados apresentados na seção anterior, chegou-se aos resultados cuja análise está dividida em duas partes. Na primeira, examinam-se os índices de ecoeficiência estimados com PPL (6) com retornos variáveis de escala. Na segunda, analisam-se os preços sombra

\subsection{RESULTADO GLOBAL}

O resultado global desta pesquisa considera a avaliação do conjunto de todas as DMU's. Observa-se que dos 3.146 municípios avaliados, somente $28 \mathrm{mu}$ nicípios, no Padrão 1, e 16 municípios, no Padrão 2, são ecoeficientes, ou seja, apresentaram um $\beta=0$, que é calculado descontando o valor de $\beta$ e as folgas em cada variável, alcançando um score $=1$. Estas unidades representam apenas $0,9 \%$ de todas as DMU's analisadas, para o Padrão 1, e 0,4\% para o Padrão 2.

Observa-se que o Padrão 2 é muito mais restritivo, menos benevolente na 
determinação das melhores práticas. Isto, como era esperado, ocorreu pelo motivo do Padrão 1 utilizar as médias estaduais para as variáveis custos fixo e variáveis.

$\mathrm{O}$ índice médio de ecoeficiência dos municípios como um todo, para o Padrão 1, é 44,6\%. Esse valor sugere que, em média, os municípios analisados podem elevar o valor total da produção (abates de suínos) e reduzir as emissões de $\mathrm{CO}_{2}$ em 44,6\% com os mesmos insumos utilizados. O índice médio de ecoeficiência dos municípios para o Padrão 2 é 53,8\%. A partir desse valor deduz-se que, em média, os municípios analisados podem elevar o valor total da produção (abates de suínos) e reduzir as emissões de $\mathrm{CO}_{2}$ em 53,8\% com os mesmos insumos utilizados.

Estes percentuais equivalem à capacidade dos municípios analisados elevarem o valor total da produção em $\mathrm{R} \$ 99.003 .950,19$, para o Padrão 1, e R\$ 130.703.236,41, para o Padrão 2.

Tabela 2. Quadro de Melhorias no Padrão 1

\begin{tabular}{l|c|c|c|c}
\hline \multicolumn{1}{c|}{ Variável } & Dados & Projeção & Metas & Diferença \% \\
\hline Custo Fixo (R\$) & 421,89 & 374,87 & $-47,02$ & $-11,1 \%$ \\
\hline Custos Variáveis (R\$) & $5.748,49$ & $5.386,21$ & $-362,28$ & $-6,3 \%$ \\
\hline Abates (R\$) & $292.784 .000,00$ & $391.787 .950,18$ & $99.003 .950,18$ & $33,8 \%$ \\
\hline $\mathrm{CO}_{2}$ (Ton) & $387.299,57$ & 244102.65 & $-143.196,91$ & $-36,9 \%$ \\
\hline
\end{tabular}

Fonte: Dados da pesquisa.

Nas Tabelas 2 e 3 revelam-se os valores absolutos das melhoras necessárias para a ecoeficiência dos municípios, para os Padrões 1 e 2 . Esses resultados foram obtidos considerando-se tanto os índices de ecoeficiência $(\beta)$ quanto as folgas (sla$c k s$ ) estimadas pelo PPL (6). Fica evidente que a economia de recursos econômicos e ambientais é substancial e o potencial de crescimento da produção não é menos importante ( $\mathrm{R} \$ 391.787 .950,18$ milhões no Padrão 1 e $\mathrm{R} \$$ 423.747.847,03 milhões, no Padrão 2). 
Tabela 3. Quadro de Melhorias no Padrão 2

\begin{tabular}{l|c|c|c|c}
\hline \multicolumn{1}{c|}{ Variável } & Dados & Projeção & Metas & Diferença \% \\
\hline Custo Fixo (R\$) & $29.111,93$ & $23.834,94$ & $-5.276,99$ & $-18,1 \%$ \\
\hline Custos Variáveis (R\$) & $402.445,75$ & $379.516,30$ & $-22.929,44$ & $-5,7 \%$ \\
\hline Abates (R\$) & $292.784 .000,00$ & $423.487 .236,40$ & $130.703 .236,41$ & $44,6 \%$ \\
\hline $\mathrm{CO}_{2}$ (Ton) & $387.299,57$ & $204.398,03$ & $-182.901,53$ & $-47,2 \%$ \\
\hline $\mathrm{CO}_{2}$ (Ton) & 56487.99 & 74814.15 & -66927.4 & $-118.48 \%$ \\
\hline
\end{tabular}

Fonte: Dados da pesquisa.

Os Estados de Mato Grosso e São Paulo são os Estados que não apresentaram nenhum município ecoeficiente, independente dos padrões. Já Mato Grosso do Sul apresentou municípios ecoeficientes somente no Padrão 1. No sentido oposto, sobressae Santa Catarina, visto que é o Estado com o maior número de municípios ecoeficientes, independente dos padrões.

Por não haver um consenso na literatura sobre uma única forma de categorização dos resultados, neste trabalho, os municípios que não atingiram um $\beta=$ 0, ou seja, não são ecoeficientes, foram classificados pelo grau de ecoineficiência. Conforme a classificação utilizada por Giacomelo e Oliveira (2014) as unidades foram categorizadas em Alta, Média ou Baixa Ecoineficiência, obtidas com o uso dos valores média e desvio padrão da ecoineficiência em cada classificação.

Os resultados do modelo demonstram que o município ecoeficiente que aparece com o maior número de vezes como referência (benchmark) para as outras DMU's, no Padrão 1, é o município goiano de Santa Bárbara de Goiás, com uma área territorial de $139,598 \mathrm{~km}^{2}$, população de 5.751 habitantes e densidade demográfica de $41,20 \mathrm{hab} / \mathrm{km}^{2}$ (Censo IBGE, 2010). Esse município está localizado na região Centro-Oeste do país e possuía um rebanho de suínos de 903 cabeças, distribuído em 61 estabelecimentos agropecuários, em 2006, apresentou uma receita com abates de $\mathrm{R} \$ 5.000,00$ por 15 cabeças abatidas (Censo Agropecuário IBGE, 2006). A variável de produto indesejado, que são as emissões de gás carbônico, apresentou um índice de $0,9135 \mathrm{TonCO}_{2}$.

Já para o Padrão 2 o município que referenciou o maior número de DMU's (benchmark) foi a cidade cearense de Palmácia, com uma área territorial de 117,813 $\mathrm{km}^{2}$, população de 12.005 habitantes e densidade demográfica de 101,90 hab/km² (Censo IBGE, 2010). Esse município está localizado na região Nordeste do país e 
possuía um rebanho de suínos de 1.410 cabeças, distribuído em 230 estabelecimentos agropecuários, apresentou uma receita com abates de $\mathrm{R} \$ 100.000,00$ por 461 cabeças abatidas, em 2006 (Censo Agropecuário IBGE, 2006). A variável produto indesejado, que são as emissões de gás carbônico, apresentou um índice de 20,3301 $\mathrm{TonCO}_{2}$.

Os municípios ecoineficientes totalizam 3.118 (99,1\% do total) no Padrão 1 e 3.131 (99,5\%) no Padrão 2, e representam o grupo constituído por todas as DMU's que não atingiram o score 1 .

No Padrão 1 o município mais distante da fronteira ecoeficiente, Herval d'Oeste (SC), possui as maiores metas, pois para atingir a ecoeficiência o município deve reduzir o produto não desejado em $96,03 \%$ e aumentar o produto desejável em $96,03 \%$. Este município obteve o maior $\beta$, apesar de estar localizado na Microrregião do Meio Oeste Catarinense (Sul do Brasil), região com grande concentração de produtores e agroindústrias suinícolas e possuía um rebanho suíno de 31.502 cabeças, distribuído em 270 estabelecimentos, com um total de 18.612 cabeças abatidas, em 2006 (Censo Agropecuário IBGE, 2006).

No Padrão 2, no município mais distante da fronteira ecoeficiente, Rosário da Limeira (MG), as metas para atingir ecoeficiência são: reduzir o custo fixo em $1,57 \%$, reduzir o produto não desejado em $89,97 \%$ e aumentar o produto desejável em $89,97 \%$. Pelo padrão 2, este município obteve o pior $\beta$. Com um rebanho suíno de 364 cabeças, distribuído em 58 estabelecimentos, um total de 43 cabeças abatidas, esse município para tornar-se ecoeficiente deve tomar como referência os municípios de Palmácia (CE), Romaria (MG) e Santa Bárbara de Goiás (GO), ou seja, deve reproduzir as práticas de uma unidade virtual formada por uma combinação linear dos pontos que representam estes três municípios.

\subsection{PREÇOS SOMBRA E O CUSTO TOTAL DAS EMISSÕES DE $\mathrm{CO}_{2}$}

As estimativas dos preços sombra das emissões de $\mathrm{CO}_{2}$, relacionadas à produção suína, foram obtidas executando o PPL (2) por duas vezes. Na primeira execução, verificou-se os índices de ecoeficiência e as melhorias necessárias, com a projeção dos municípios ecoineficientes à fronteira. Na segunda execução, após corrigidas as projeções, estimou-se os preços sombra, que representam a taxa marginal de transformação entre os abates (saídas desejáveis) e as emissões de $\mathrm{CO}_{2}$ (saídas indesejáveis) (ARANDIA; ALDANONDO-OCHOA, 2011). 
Desta forma, os preços sombra relativos são aferidos pela inclinação da tangente na projeção na fronteira eficiente do CPP, ou seja, a partir da taxa marginal de transformação dos produtos envolvidos ( $b$ e $y$ ). Assim, indicam a receita marginal com os abates que cada unidade produtiva ecoeficiente tem que renunciar para reduzir as emissões de carbono (ARANDIA; ALDANONDO-OCHOA, 2011).

Nos resultados da Tabela 4 pode-se evidenciar que nenhum dos valores foi positivo, o que aponta para a incompatibilidade da redução das emissões com o aumento da produção, na ecoeficiência. Isto indica que a fronteira do conjunto de possibilidade de produção (CPP) tem a forma representada na Figura 1 e que, com ecoeficiência, a redução das emissões é incompatível com a ampliação da produção.

Tabela 4. Preço sombra médio do $\mathrm{Co}_{2}$ dos abates de suínos

\begin{tabular}{lcc}
\hline \multicolumn{1}{c}{ Estado } & $\begin{array}{c}\text { Preço Sombra Médio } \\
\text { (R\$) Padrão 1 }\end{array}$ & $\begin{array}{c}\text { Preço Sombra Médio } \\
\text { (R\$) Padrão 2 }\end{array}$ \\
\hline Ceará & $-4.306,58$ & $-2.794,06$ \\
Goiás & $-1.746,73$ & $-1.473,46$ \\
Mato Grosso & $-1.824,02$ & $-804,21$ \\
Mato Grosso do Sul & $-977,40$ & $-816,96$ \\
Minas Gerais & $-2.539,60$ & $-2.477,96$ \\
São Paulo & $-3.009,41$ & $-2.411,59$ \\
Paraná & $-1.382,49$ & $-1.215,95$ \\
Santa Catarina & $-1.464,21$ & $-1.358,18$ \\
Rio Grande do Sul & $-1.772,43$ & $-1.399,93$ \\
Totalização de todos os municípios & $-3.539,81$ & $-3.677,07$ \\
\hline
\end{tabular}

Fonte: Dados da pesquisa.

É necessário destacar também que, por um lado, os municípios com baixos níveis de emissões têm tangentes mais inclinadas e tendem a apresentar preços sombra elevados, confirmando que o produtor nesta situação tem que realizar um esforço maior para reduzir as mesmas. Por outro lado, os municípios com altos níveis de emissões exibem inclinação decrescente e preços sombra menores. Isto indica que eles têm custos baixos para reduzi-las. Assim, o Padrão 1, conforme a Tabela 6 indica que Ceará é o Estado com maior média no preço sombra dentro do conjunto dos 
Estados com municípios produtores de suínos. Isto fica óbvio quando se observa que a projeção na fronteira de muitos municípios deste Estado sugere níveis relativamente baixos de emissões. Pelo contrário, Mato Grosso do Sul é o Estado com a menor média no preço sombra. A explicação está no fato deste Estado apresentar o maior nível de emissões em relação ao nível de produção. O Padrão 2 revela novamente o Ceará como o Estado com maior média no preço sombra e Mato Grosso e Mato Grosso do Sul como os Estados com as menores médias no preço sombra. $\mathrm{O}$ preço sombra médio de todos os municípios ficou em $\mathrm{R} \$ 3.539,81$ no Padrão 1 e $\mathrm{R} \$$ 3.677,07 no Padrão 2.

Partindo desses resultados pode-se, ainda, estimar o custo para reduzir as emissões dos abates, através da multiplicação do preço sombra pelas emissões de $\mathrm{CO}_{2}$. Este valor representa o custo para produzir sem emitir GEEs.

Conforme a Tabela 5, este custo para ambos os padrões, se concentra no do Rio Grande do Sul, pois esse Estado possui o maior Fator de Emissão de Metano no Manejo de Dejetos, conforme Tabela 1.

Tabela 5. Custos da redução das emissões de $\mathrm{CO}_{2}$

\begin{tabular}{|c|c|c|}
\hline Estado & $\begin{array}{c}\text { Custo Médio da Redução de Emis- } \\
\text { sóes de } \mathrm{CO}_{2}(\mathrm{R} \$) \\
\text { Padrão } 1\end{array}$ & $\begin{array}{c}\text { Custo Médio da Redução de } \\
\text { Emissões de } \mathrm{CO}_{2}(\mathrm{R} \$) \\
\text { Padrão } 2\end{array}$ \\
\hline Ceará & $18.585 .466,92$ & $19.306 .148,20$ \\
\hline Goiás & $107.964 .632,26$ & $112.151 .133,98$ \\
\hline Mato Grosso & $65.861 .474,42$ & $68.415 .358,69$ \\
\hline Mato Grosso do Sul & $34.451 .053,57$ & $35.786 .948,41$ \\
\hline Minas Gerais & $191.502 .354,99$ & $198.928 .165,85$ \\
\hline São Paulo & $51.466 .045,91$ & $53.461 .724,35$ \\
\hline Paraná & $242.694 .985,61$ & $252.105 .872,81$ \\
\hline Estado & $\begin{array}{c}\text { Custo Médio da Redução de Emis- } \\
\text { sões de } \mathrm{CO}_{2}(\mathrm{R} \$) \\
\text { Padrão } 1\end{array}$ & $\begin{array}{c}\text { Custo Médio da Redução de } \\
\text { Emissões de } \mathrm{CO}_{2}(\mathrm{R} \$) \\
\text { Padrão } 2\end{array}$ \\
\hline Santa Catarina & $156.702 .851,37$ & $162.779 .255,68$ \\
\hline Rio Grande do Sul & $501.738 .020,88$ & $521.193 .717,10$ \\
\hline $\begin{array}{l}\text { Totalização de todos os } \\
\text { municípios }\end{array}$ & $1.370 .966 .885,93$ & $1.424 .128 .325,06$ \\
\hline
\end{tabular}

Fonte: Dados da pesquisa. 
Para toda a amostra, o valor no Padrão 1 é de R\$1,37 bilhões, e no Padrão 2 de $R \$ 1,42$ bilhões. O primeiro equivale a $468 \%$ do valor da produção do ano estudado, o segundo representa 486\%. Percebe-se que a incorporação desses custos é praticamente inviável, levaria à quebra as propriedades.

Contudo, como os pagamentos por serviços ambientais são uma das alternativas de compensação, pode-se redesenhar um sistema dissuasivo de pagamentos e compensação que puna os poluidores e recompense os produtores que reduzam as emissões ou utilizem tecnologias mais limpas. Esse sistema pode usar os preços sombras como referência.

\section{CONSIDERAÇÕES FINAIS}

O objetivo deste trabalho foi estimar a ecoeficiência na produção brasileira de suínos, os preços sombra e o custo total das emissões de Gases de Efeito Estufa (GEE) do setor, utilizando a Análise Envoltória de dados (DEA) combinado com o método de Funções Distâncias Direcionais (DDF) nos municípios de nove Estados produtores. Desse modo, contribui de forma importante no que diz respeito às pesquisas relacionadas à ecoeficiência, aos preços sombra e aos custos de oportunidades de externalidades negativas na suinocultura brasileira, as quais ainda são escassas na literatura.

O cálculo da ecoeficiência dos municípios com abates de suínos nos nove Estados pesquisados ocorreu conforme a metodologia e dados apresentados e permitiu estimar em quanto é possível aumentar a produção e reduzir a emissão de gases de efeito estufa nos abates, simultaneamente, com a manutenção dos mesmos custos na produção. Foi verificado que dos 3.146 municípios avaliados, apenas 28 foram ecoeficientes no padrão 1 e 16 foram ecoeficientes no padrão 2 , com um índice médio de ecoeficiência dos municípios de 0,4467 no Padrão 1 e de 0,5389 no Padrão 2.

Em valores reais, para a suinocultura ser mais ecoeficiente, os municípios podem elevar o valor total dos abates de suínos em $\mathrm{R} \$ 99$ milhões e reduzir as emissões de $\mathrm{CO}_{2}$ em 143 mil toneladas, com os mesmos insumos, no Padrão 1. No 
padrão 2, esses valores seriam de $\mathrm{R} \$ 130$ milhões na receita dos abates e 182 mil toneladas de redução de emissões de $\mathrm{CO}_{2}$, com os mesmo insumos.

Com base nisso, para validar a questão do problema de pesquisa, podemos concluir que as questões ambientais e econômicas não são antagônicas e não resultam em um jogo de soma zero. Possui uma relação win-win, ou seja, todos ganham.

Portanto, a pesquisa demonstra que o setor suinícola brasileiro apresentou compatibilidade entre a eficiência econômica e as limitações dos recursos naturais, pois pode aumentar os ganhos na produção e, simultaneamente, reduzir as emissões de GEEs. E essas melhorias podem ocorrer apenas copiando as melhores práticas nacionais.

Os resultados dos preços sombras das emissões dos abates de suínos nos municípios avaliados indicaram que os custos da poluição são significativos nos Estados com um menor nível de intensificação da produção. O preço sombra médio foi de R\$3.539,81 no Padrão 1 e R\$ 3.677,07 no Padrão 2.

Eles permitiram estimar também o custo total da redução das emissões dos abates, que resultaram aproximadamente em $\mathrm{R} \$ 1,3$ bilhões em ambos os padrões. Em 2006, o valor total da produção foi de R\$ 292 milhões. No Padrão 1 equivale a $463 \%$ do valor da produção do ano estudado, no Padrão 2 representa 478\%.

Portanto, o custo estimado da redução das emissões de $\mathrm{CO}_{2}$ demonstra que a incorporação dos mesmos é praticamente inviável, pois os retornos econômicos não suportam a internalização desses custos e levaria à quebra das propriedades.

Uma forma de solucionar essa questão seria a criação de um sistema que tome em consideração os preços sombra, através de compensações para as ações que reduzam as emissões ou utilizem tecnologias mais limpas, para que os produtores de suínos internalizem as externalidades negativas.

Quanto às limitações desta pesquisa, cabe advertir que os índices e os preços sombra estimados consideram apenas a tecnologia de produção, ou seja, desconsideram a capacidade do planeta de absorver e suportar as emissões de $\mathrm{CO}_{2}$. Neste sentido, subestimam o custo real do impacto ambiental. Mesmo assim, tendo em conta as incertezas associadas à irreversibilidade dos crescentes impactos ambientais, a pesquisa indica a necessidade de redesenhar um sistema de sanções e incentivos. 
Para finalizar, cabe destacar também que existe um grande potencial de pesquisa no que se refere à eficiência da suinocultura no Brasil, já que são poucos os estudos com esse enfoque. Novos estudos podem analisar a dinâmica do desempenho econômico ambiental do setor. A introdução de séries temporais permitirá criar um modelo dinâmico, que desloca a questão central para outros problemas muito importantes: 1) a evolução da ecoeficiencia de cada unidade relativamente à evolução do conjunto de unidades avaliadas; 2) a decomposição do índice de progresso da ecoeficiência em termos de mudanças tecnológicas, eficiência técnica pura e de escala; e 3) a natureza da trajetória temporal, com ou sem flutuações, com tendência a convergir ou divergir.

\section{REFERÊNCIAS}

ABCS. MAPA. EMBRAPA. Manual Brasileiro de Boas Práticas Agropecuárias na Produção de Suínos. Brasília: ABCS; MAPA; Concórdia: Embrapa Suínos e Aves. 140 p. 2011.

ARANDIA, A.; ALDANONDO-OCHOA, A. Pollution shadow prices in conventional and organic farming: an application in a Mediterranean context. Spanish Journal of Agricultural Research, v. 9, n. 2, p. 363-376, 2011

CALLENS, I.; TYTECA, D. Towards Indicators of Sustainable Development for Firms: Concepts and Definitions. Ecological Economics, v. 28, p. 41-53, 1999. DOI: 10.1016/S0921-8009(98)00035-4.

CAMPOS, S. A. C.; COELHO, A. B.; GOMES, A. P.; MATTOS, L. B. Eficiência e custos associados à adequação ambiental para a produção láctea em Minas Gerais. Organizações Rurais \& Agroindustriais, v. 16, n. 3, p. 324-342, 2014.

CASTRO, J. D. B. Usos e abusos da valoração econômica do meio ambiente: ensaios sobre aplicações de métodos de função demanda no Brasil. 2015. $250 \mathrm{f}$. Tese (Doutorado em Economia) Universidade de Brasília, Brasília, 2015.

CATALÁN, P.; DESTÉFANO, O. ¿Cuál es el costo de la contaminación ambiental minera sobre los recursos hídricos en el Perú? Lima: Pontificia Universidad Católica del Perú. Departamento de Economía, 2011. 
CHUNG, Y.; FÄRE, R.; GROSSKOPF, S. Productivity and undesirable outputs: a directional function approach. Journal Environ. Manag., v. 51, p. 229-240, 1997.

COGGINS, J. S.; SWINTON, J. R. The price of pollution: A dual approach to valuing SO2 allowances. Journal of Environmental Economics and Management, v. 30. p. 58-72, 1996.

COSER, F. J. Contrato de integração de suínos: formatos, conteúdos e deficiências da estrutura de governança predominante na suinocultura brasileira. 2010. 160f. Dissertação (Mestrado) - Faculdade de Agronomia e Medicina Veterinária, Universidade de Brasília, UnB, Brasília, 2010.

FÄRE, R.; GROSSKOPF, S.; TYTECA, D. An activity analysis model of the environment performance of firms: application to fossil-fuel-fired electric utilities. Ecol. Econ., v. 18, p. 161-175, 1996.

FÄRE, R.; GROSSKOPF, S.; WEBER, W. L. Shadow prices and pollution costs in U. S. agriculture. Ecological Economics, v. 56, n. 1, p. 89-103, 2006.

FARRELL, M. J. The Measurement of Productive Efficiency. Journal of the Royal Statistical Society, v. 120, n. (part III), p. 253-289, 1957.

FÁVERO, J. A. Produção suínos. Apostila sistema de produção de suínos. Embrapa Suínos e Aves, jul. 2003.

FREEMAN, M. A.; HAVEMAN, R. H.; KNEESE, A. V. The Economics of Environmental Policy. John Wiley \& Sons, New York, 1973.

GASTARDELO, T. A. R.; MELZ, L. J. A suinocultura industrial no mundo e no Brasil. Revista UNEMAT de Contabilidade, v. 3, n. 6, 2014.

GIACOMELO, C. P.; OLIVEIRA, R. L. Análise Envoltória de Dados (DEA): uma Proposta para Avaliação de Desempenho de Unidades Acadêmicas de Uma Universidade. Revista Gestão Universitária na América Latina (GUAL), Florianópolis, v. 7, n. 2, p. 130-151, mai. 2014.

GOMES, E. G.; MANGABEIRA, J. A. C. Uso de análise de envoltória de dados em agricultura: O caso de Holambra. Engevista, v. 6, n. 1, p. 19-27, abr. 2004. 
GOMES, E. G.; MELLO, J. C. C. B. S.; MANGABEIRA, J. A. C. Estudo da sustentabilidade agrícola em município amazônico com análise envoltória de dados. Pesqui. Oper., v. 29, n. 1, Jan./Apr. 2009.

GROSSKOPF, S. The Role of the Reference Technology in Measuring Productive Efficiency. The Economic Journal, 96, p. 499-513, June, 1986.

INSTITUTO BRASILEIRO DE GEOGRAFIA E ESTATÍSTICA - IBGE. Sistema IBGE de recuperação automática - SIDRA. 2015.

INSTITUTO BRASILEIRO DE GEOGRAFIA E ESTATÍ́STICA - IBGE. Pesquisa Trimestral do Abate de Animais. 2011.

IPCC. Climate Change 1995 - The Science of Climate Change: Summary for Policymakers and Technical Summary of the Working Group I Report. Intergovernmental Panel on Climate Change, Cambridge, 1996.

MAGALHÃES, K.; CAMPOS, R. Eficiência técnica e desempenho econômico de produtores de leite no Estado do Ceará, Brasil. RER, Rio de Janeiro, v. 44, n. 4, p. 695 711, out./dez. 2006.

MAPA. MINISTÉRIO DA AGRICULTURA, PECUÁRIA E ABASTECIMENTO. Projeções do Agronegócio Brasil - 2014/2015 a 2024/2025. 2015.

MARIANO, E. B. Sistematização e comparação de técnicas, modelos e perspectivas não-paramétricas de análise de eficiência produtiva. 2008. 280f. Dissertação (Mestrado)- Escola de Engenharia de São Carlos, EESC USP, São Carlos, 2008.

MAZOYER, M.; ROUDART, L. História das agriculturas no mundo: do neolítico à crise contemporânea. São Paulo: Edunesp; DF: NEAD, 2010.

MCT. Ministério da Ciência e Tecnologia. Emissões de Metano por Fermentação Entérica e Manejo de Dejetos de Animais. Relatórios de Referência: Agricultura. $2^{\circ}$ Inventário Brasileiro de Emissões e Remoções Antrópicas de Gases de Efeito Estufa. Brasília: MCT, 2010.

MIELE, M. Contratos, especialização, escala de produção e potencial poluidor na suinocultura de Santa Catarina. 2006. 278f. Tese (Doutorado) - Programa de 
Pós-Graduação em Agronegócios, Centro de Estudos e Pesquisas em Agronegócios, Universidade Federal do Rio Grande do Sul, Porto Alegre, 2006.

MIELE, M.; ALMEIDA, M. M. T. B.; MONTICELLI, C. J.; OLIVEIRA, O. C.; BOFF, J. A.; PALHARES, J. C. P.; SANDI, A. J.; CARDOSO, L. S. Caracterização da suinocultura no Brasil a partir do censo agropecuário 2006 do IBGE. Concórdia: Embrapa Suínos e Aves, 2013. 149 p.

MOTA, J.A. O valor da natureza: Economia e Política dos recursos naturais. Rio de Janeiro: Gramond, 2001.

PADRÃO, G. A. et al. Environmental efficiency and opportunity cost of the Forest Code for the Amazon. RIO+20 - International Society for Ecological Economics Conference. Rio de Janeiro: [s. n.]. 2012.

PICAZO-TADEO, A. J.; BELTRÁN-ESTEVE, M.; GÓMEZ-LIMÓN, J. A. Assessing eco-efficiency with directional distance functions. European Journal of Operational Research, v. 220, n. 3, p. 798-809. 2012.

PICAZO-TADEO, A. J.; GÓMEZ-LIMÓN, J. A.; REIG-MARTÍNEZ, E. Assessing farming eco-efficiency: a data envelopment analysis approach. Journal of Environmental Management, n. 92, p. 1154-1164. 2011.

ROSANO-PEÑA, C. Um Modelo de Avaliação da Eficiência da Administração Pública através do Método Análise Envoltória de Dados (DEA). RAC, Curitiba, v. 12, n. 1, p. 83-106, Jan./Mar. 2008.

ROSANO-PENAA, C.; DAHER, C. E.; MEDEIROS, O. R. Ecoeficiência e Impacto da Regulação Ambiental na Agropecuária Brasileira com Funções Distância Direcionais. In: ENCONTRO DA ANPAD, 37., 2013. Anais [...]. Rio de Janeiro, 2013.

ROSANO-PEÑA, C.; GUARNIERI, P.; SOBREIRO, V. A.; SERRANO, A. L. M.; KIMURA, $\mathrm{H}$. A medida de sustentabilidade do agronegócio brasileiro usando funções de distância direcionais e análise envoltória de dados. Revista Internacional de Desenvolvimento Sustentável e Ecologia Mundial, v. 21, p. 210-222, 2014.

SALNYKOV, M. I.; ZELENYUK, V. P. Estimation of environmental efficiencies of economies and shadow prices of pollutants in countries in transition. Mos- 
cow: EERC, 2005.

SANTOS, J.; TALAMINI, D.; MARTINS, F. Distribuição espacial da produção de suínos no Brasil. 2011.

SARDÁ, L. G.; HIGARASHI, M. M.; MULLER, S.; OLIVEIRA, P. A.; COMIN, J. J. et al. Redução da emissão de $\mathrm{CO}_{2}, \mathrm{CH}_{4}$ e $\mathrm{H}_{2} \mathrm{~S}$ através da compostagem de dejetos suínos. Rev. bras. eng. agríc. ambient, Campina Grande, v. 14, n. 9, 2010.

SILVA, C. L.; BASSI, N. S. S.; NASCIMENTO, D. E. A implementação de políticas públicas pelas instituições públicas de pesquisa: um estudo sobre pesquisas e tecnologias da Embrapa Suínos e Aves para mitigação do impacto ambiental da suinocultura no oeste catarinense. Revista Espacios, Caracas, v. 32, n. 3, p. 9-10, 2011.

SOUZA, G. S.; ALVES, E.; GOMES, E. G. Pesquisa, extensão e políticas públicas na agricultura brasileira. Revista de Política Agrícola, v. 23, n. 2, p. 69-76, 2014.

TYTECA, D. On the measurement of the environmental performance of firms - a literature review and a productive efficiency perspective. Institut d'Administration et de Gestion, Université Catholique de Louvain, Place des Doyens, Louvain-la-Neuve, Belgium. Journal Environmental Economics and Management, 46, p. 281-308. 1996.

USDA. Livestock and Poultry: World Markets and Trade. World Production, Markets, and Trade Reports. Foreign Agricultural Service. United States Departament Agriculture, 2015.

ZHANG, B.; BI, J.; FAN, Z.; YUAN, Z.; GE, J. Eco-efficiency analysis of industrial system in China: A data envelopment analysis approach. Ecological Economics, v. 68, n. 1-2, p. 306-316, 2008.

ZHOU, P.; ANG, B. W.; POH, K. L. A survey of data envelopment analysis in energy and environmental studies. Department of Industrial and Systems Engineering, National University of Singapore, Singapure. European Journal of Operational Research, 189, p. 1-18, 2008. 